SLAC-PUB-8250

September 1999

\title{
Split Fermions in Extra Dimensions and Exponentially Small Cross-Sections at Future Colliders
}

N. Arkani-Hamed et al.

Submitted toJournal of High Energy Physics (JHEP)

Stanford Linear Accelerator Center, Stanford University, Stanford, CA 94309

Work supported by Department of Energy contract DE AC03 76SF00515. 
UCB-PTH-99/41

LBNL-44254

SLAC-PUB-8250

September 1999

\title{
Split Fermions in Extra Dimensions and Exponentially Small Cross-Sections at Future Colliders
}

\author{
Nima Arkani-Hamed, ${ }^{a *}$ Yuval Grossman ${ }^{b \dagger}$ and Martin Schmaltz ${ }^{b \ddagger}$ \\ ${ }^{a}$ Department of Physics, University of California, Berkeley, CA 94720 \\ and Theoretical Physics Group, LBNL, University of California, Berkeley, CA 94720. \\ ${ }^{b}$ Stanford Linear Accelerator Center \\ Stanford University, Stanford, CA 94309
}

\begin{abstract}
We point out a dramatic new experimental signature for a class of theories with extra dimensions, where quarks and leptons are localized at slightly separated parallel "walls" whereas gauge and Higgs fields live in the bulk of the extra dimensions. The separation forbids direct local couplings between quarks and leptons, allowing for an elegant solution to the proton decay problem. We show that scattering cross sections for collisions of fermions which are separated in the extra dimensions vanish exponentially at energies high enough to probe the separation distance. This is because the separation puts a lower bound on the attainable impact parameter in the collision. We present cross sections for two body high energy scattering and estimate the power with which future colliders can probe this scenario, finding sensitivity to inverse fermion separations of order 10-70 TeV.
\end{abstract}

\footnotetext{
*arkani@thsrv.1bl.gov

†yuvaleslac.stanford.edu

${ }^{\ddagger}$ schmaltz@slac.stanford.edu
} 


\section{INTRODUCTION}

Any extension of the Standard Model (SM) with a low fundamental scale $M_{*}$ has to explain why it does not predict rapid proton decay through higher dimensional operators suppressed by $M_{*}$. In the SM proton decay is not a problem as the lowest-dimensional baryon number violating operator is dimension six and is harmless when suppressed by the enormous value of the Planck mass. However this becomes a serious problem in theories which attempt to nullify the hierarchy between the Planck scale and the weak scale by postulating that the fundamental scale of gravity really is at or near a TeV, and that the apparent weakness of gravity is due to a large extra-dimensional volume into which the gravitational field can spread [1]-1 $\left[\begin{array}{l}1 \\ -1\end{array}\right]$.

One approach to this problem is to forbid proton decay by postulating a new symmetry. This symmetry would have to be gauged as gravitational effects involving virtual black holes and worm holes violate global symmetries and generate dangerous $M_{*}$-suppressed proton decay operators. But anomaly cancellation conditions for gauge symmetries make it difficult to find consistent theories. The only known example is baryon triality [ịi] which stabilizes the proton but has baroque charge assignments.

A different solution to the proton decay problem which does not rely on symmetries but rather exploits the new space in the extra dimensions was proposed in Ref. [i is to separate quarks from leptons in the extra dimensions. Consider for example a model where the SM gauge and Higgs fields live in the bulk of one extra compact dimension of radius $\mathrm{TeV}^{-1}$ while the quarks and leptons are localized at different positions with narrow wavefunctions in the extra dimension. . $_{-1}^{\prime}$ This separation of the fermion fields suppresses proton decay because direct couplings of quarks to leptons are forbidden by five dimensional locality; the proton decay rate is exponentially suppressed by the overlap of the quark and lepton wavefunctions.

At low energies $(E \ll \mathrm{TeV})$, experiments cannot resolve the size of the extra dimension and its substructure. One observes fermions coupled to the lightest modes of the gauge fields with couplings exactly as in the SM. Experiments at energies above TeV would discover a whole tower of Kaluza Klein (KK) excitations of the gauge and Higgs fields, proving that Higgs and gauge fields propagate in the bulk of an extra dimension. Measurements of the

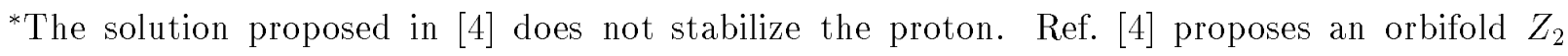
symmetry which does not allow GUT gauge interactions to contribute to proton decay. However, this symmetry does not forbid any of the fatal higher dimensional operators such as $Q Q Q L$ which lead to disastrous proton decay. These operators are the major problem for theories with a low fundamental scale, even in the absence of GUT symmetries.

†The gauge fields may also be confined to a brane of thickness $\mathrm{TeV}^{-1}$ in much larger extra dimensions. Then the fermions would be stuck to thin parallel "layers" within the brane.
} 
couplings of the various KK fields to the fermions can be used to map out the locations of the quarks and leptons in the extra dimensions. But even at lower energies virtual KK mode exchange leads to small deviations in precision measurements. For example, as shown

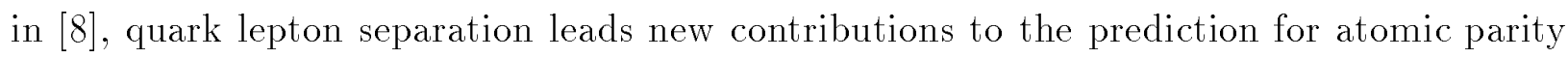

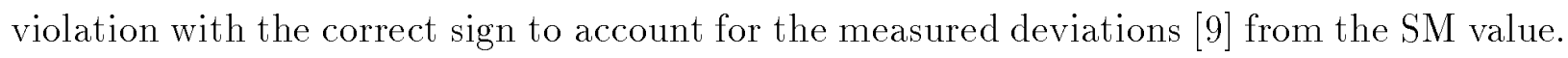

Even though we motivate the quark-lepton separation from proton decay we note that fermion separation can be more general with all fermions separated in the extra dimensions. In any realistic model the locations of all the fermion fields are determined by potentials which depend on the various parameters of the theory. Since the different SM fermion fields have different gauge and Yukawa couplings we expect their potentials to differ, leading to splittings in their positions.

In this paper we point out a dramatic and model independent experimental signature of this scenario which follows simply from locality in the extra dimensions: At energies above a $T e V$, the large angle scattering cross section for fermions which are separated in the extra dimensions falls off exponentially with energy. This is easily understood from the fact that the fermion separation in the extra dimensions implies a minimum impact parameter of order $\mathrm{TeV}^{-1}$. At energies corresponding to shorter distances the large angle cross section falls off exponentially because the particles "miss" each other. The amplitude involves a Yukawa propagator for the exchanged gauge boson where the four dimensional momentum transfer acts as the mass in the exponential. More precisely, we find exponential suppression in any $t$ and $u$ channel scattering of split fermions. However, $s$ channel exchange is timelike, and therefore the fermion separation in space does not force an exponential suppression. Nevertheless, $s$ channel processes also lead to interesting signatures as the interference of the SM amplitude with KK exchange diagrams depends on the fermion separation.

The remainder of this paper is structured as follows: Section 2 reviews the basic setup and explains how quark lepton separation suppresses proton decay. In Section 3 we develop the necessary formulae to calculate scattering cross sections in our framework. In Section 4 we apply the results of section 3 to different physical systems (deep inelastic scattering, $e^{+} e^{-}$and $\mu^{+} \mu^{-}$scattering) and show the reach and physics potential of various colliders. Section 5 contains final discussion.

\section{FRAMEWORK: EXTRA DIMENSIONAL GEOGRAPHY}

In this section we describe our framework and review the ideas which lead to it. Our starting point is the observation that simple compactifications of higher dimensional theories typically do not lead to chiral fermions. The known mechanisms which do lead to chiral spectra usually break translation invariance in the extra dimensions and the chiral fermions are localized at special points in the compact space. Examples include twisted sector fermions stuck at orbifold fixed points in string theory, chiral states from intersecting D-branes, or zero modes trapped to defects in field theory. Given that fermions generically 
are localized at special points in the extra dimensions we are motivated to consider the possibility of having different locations for the different SM fermions. In such a scenario locality in the higher dimensions forbids direct couplings between fermions which live at different places. This suppression of contact terms between fermions is very generic and leads to approximate symmetries in the effective four dimensional theory. In our framework the observed approximate global symmetries of the SM (such as baryon $(B)$ and lepton $(L)$ number) are not accidental, they follow from non-trivial geography in the extra dimensions. The gauge and Higgs fields are necessarily bulk fields because they need to couple to all the SM fermions. Gauge and Higgs field exchange does generate non-local interactions but the effective operators obtained in this way preserve $B$ and $L$ and cannot lead to proton decay.

Let us discuss corrections to the above picture in detail. There are two possible sources of interactions between quarks and leptons: direct local couplings (contact terms), or quarks and leptons could both couple to a new de-localized "bulk" field which would act as messenger and lead to couplings which are non-local in the extra dimension.

Direct local interaction require the wave functions of quarks and leptons to overlap. The resulting effective four dimensional coupling is proportional to this overlap. If, as in the

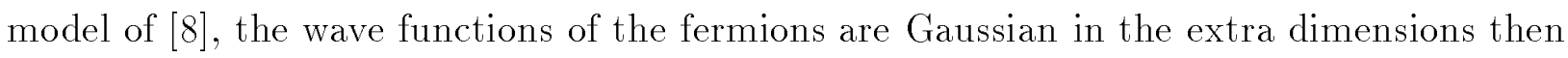
the effective four dimensional couplings are Gaussian in the distance between quarks and leptons. A quark-lepton separation of 8 in units of the fermion wave function's width leads to a factor $\sim \exp (-50)$ which suppresses proton decay to safety.

What about non-local interactions via bulk messengers? Generating proton decay requires a bulk messenger with $B$ and $L$ violating couplings. In addition, this messenger has to be a fermion as the proton's fermion number has to be transferred to the final state leptons. If the theory does contain a bulk fermion with $B$ and $L$ violating couplings, we can estimate the strength of the resulting effective proton decay operator. The relevant Feynman diagram (in position space) involves the Yukawa propagator of the messenger field from the quarks in one location in the extra dimension to the leptons. For a messenger of mass $M$ and a quark-lepton separation $d$ the propagator contains an exponential $\exp (-M d)$. Thus, in order to avoid the proton decay bounds we require that all bulk fermions with $B$ and $L$ violating couplings be heavier than the inverse quark-lepton separation by a factor of about 50. Note that even much lighter bulk fermions can be harmless if $B-L$ is imposed as a gauge symmetry. Then the messenger fermion also needs to carry the $B-L$ charge of the proton in order to be dangerous.

While it will not be of central importance for this paper we would like to mention a particularly satisfying picture for the origin of the fermion separations in the context of fermion zero modes stuck at defects: Assume that the SM is unified into $S O(10)$ in the fivedimensional theory at energies near $\sim 10 \mathrm{TeV}$. Then splitting between quarks and leptons at lower energies has a natural explanation if the fermion fields' localization potential contains terms which couple to a GUT symmetry breaking vacuum expectation value in the $B-L$ direction [i] 
We note that in addition to quark-lepton separation there may also be splittings between the generations. The separation of left- and right-handed components of the SM quarks and leptons could then explain the hierarchies in the SM Yukawa couplings [i] The separations needed to produce realistic quark and lepton masses are in the range (0..5) in units of the wave-function width in the case of Gaussian wave functions [isi]. Explicit examples that reproduce the observed femion masses were worked out in [i]1].

Let us summarize the scales involved in the theory. The lowest experimentally allowed

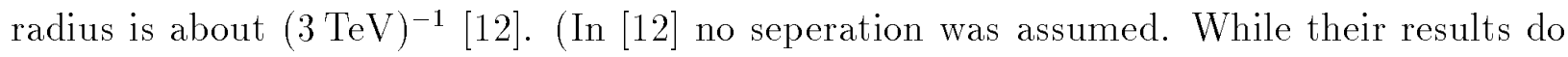
not directly apply to our case, the order of magnitude of the bounds should be the same.) At energies above a few $\mathrm{TeV}$ the theory becomes effectively higher dimensional, but we can continue to use a four dimensional description by including KK excitations for the bulk gauge and Higgs fields. The loop expansion parameter in this effective theory is

$$
\frac{g^{2} N_{K K}}{16 \pi^{2}}
$$

where $g$ stands for any of the SM gauge couplings and $N_{K K}$ is the number of KK excitations contributing in the loop. Our perturbative description of physics breaks down when this

parameter is of order unity which occurs for $N_{K K} \sim 100$ or $M_{*} \sim 100 \mathrm{TeV}$. The width of the fermion wave functions in the extra dimension is more model dependent. In the field theoretic construction of [i] it must be at least a factor of 10 narrower than the separation in order to sufficiently suppress proton decay.

It should be clear from this discussion that the scale of quark-lepton separation is well below the scale where the theory becomes strongly coupled, and where quantum gravity or stringy effects may become important. The fermion separation serves as an energy cut-off and suppresses incalculable high energy contributions from the unknown theory of quantum gravity.

\section{SCATTERING OF FERMIONS LOCALIZED AT DIFFERENT PLACES}

\section{A. One extra dimension}

Let us now imagine colliding fermions which are localized at two different places in a circular extra dimension of radius $R$. Motivated by the solution to the proton decay problem discussed above, we will begin by considering the scattering of electrons on protons, although we can imagine more generally that any set of the $\left(Q, U^{c}, D^{c}, L, E^{c}\right)$ fields are split in the extra dimensions; indeed our most interesting experimental signatures will be for the case of separations in the lepton sector.

In the context of our model there are three potentially relevant mass scales for this collision: the momentum transfer of the $t$-channel scattering $\sqrt{-t}$, the inverse of the quarklepton separation $d^{-1}$ which we take to be of order of the inverse thickness $R^{-1}$ of the 
extra dimension, and the inverse width of the fermion wave functions $\sigma^{-1}$. However, as discussed above proton stability requires quarks to be well separated from leptons and we will approximate the fermion wave functions by delta functions for the calculation. At the end of this section we will compute the corrections which arise from the finite width of the wave functions and verify that they are negligible for practical purposes.

To calculate the scattering though intermediate bulk gauge fields we can either choose to work with a five-dimensional propagator directly or else add contributions from an infinite tower of KK excitations in a four dimensional context. It is instructive to do it both ways. The five dimensional propagator in momentum space is $\left(t-p_{5}^{2}-m^{2}\right)^{-1}$ where we separated out the five dimensional momentum transfer $p_{5}$. As we are interested in propagation between definite positions in the fifth dimension it is convenient to Fourier transform in the fifth coordinate

$$
P_{d}(t)=\sum_{n=-\infty}^{\infty} \frac{e^{i n d / R}}{t-(n / R)^{2}-m^{2}},
$$

where $d=x_{q}-x_{l}$ and $x_{f}$ is the location of fermion $f$ in the extra dimension. The Fourier transform is a sum and not an integral since momenta in the fifth coordinate are quantized in units of $1 / R$.

This propagator can also be understood in the four dimensional (4d) language as arising from exchange of the $4 \mathrm{~d}$ gauge boson and its infinite tower of KK excitations. To see this expand the KK excitations of the gauge field in plane waves, $\exp \left(\right.$ in $\left.x_{5} / R\right)$. Each of these KK modes has a four dimensional propagator $\left(t-(n / R)^{2}-m^{2}\right)^{-1}$. Furthermore, the couplings to the fermions differ for the various KK gauge bosons. They follow from expanding the five dimensional action

$$
\int d x_{5} d^{4} \mathbf{x} \delta\left(x_{5}-x_{f}\right) g \bar{\Psi}(\mathrm{x}) \mathcal{A}\left(\mathrm{x}, x_{5}\right) \Psi(\mathrm{x})=\int d^{4} \mathrm{x} \sum_{n} g e^{i n x_{f} / R} \bar{\Psi}(\mathrm{x}) \mathcal{A}^{n}(\mathrm{x}) \Psi(\mathrm{x}) .
$$

Thus the modified couplings are $g_{n}=g e^{i n x_{f}} / R$. We can now write the "KK-tower propagator" which is a sum over the propagators of the KK modes, including phase factors from the

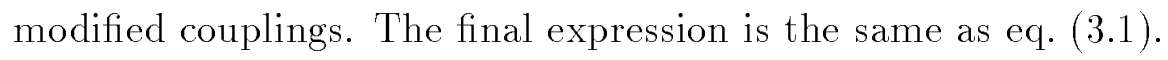

This propagator can be simplified by performing the sum. To this end one rewrites it as a contour integral with a cigar-shaped contour that encircles the real axis and then deforming the contour

$$
P_{d}(t)=\oint \frac{d n}{2 \pi i} \frac{\pi}{\sin (\pi n)} \frac{e^{i n(d / R-\pi)}}{t-(n / R)^{2}-m^{2}} .
$$

Performing the integral we find

$$
P_{d}(t)=-\frac{\pi R}{\sqrt{-t+m^{2}}} \frac{\cosh \left[(d-\pi R) \sqrt{-t+m^{2}}\right]}{\sinh \left[\pi R \sqrt{-t+m^{2}}\right]} .
$$

The Feynman rules for diagrams involving exchange of bulk gauge fields are now identical to the usual four dimensional SM Feynman rules except for the replacement of $4 \mathrm{~d}$ gauge boson 
propagators by the corresponding $5 \mathrm{~d}$ propagators. Before we proceed with calculating cross sections we note a few properties of the propagator we just found.

It is easy to understand the two limits $\sqrt{-t} \gg R^{-1}$ and $\sqrt{-t} \ll R^{-1}$. In the former case we obtain

$$
P_{d}(t) \simeq-\frac{\pi R}{\sqrt{-t}} e^{-\sqrt{-t} d},
$$

which vanishes exponentially with the momentum transfer in the process as we anticipated from five dimensional locality. In the limit of small momentum transfer we obtain

$$
P_{d}(t) \simeq \frac{1}{t-m^{2}}-R^{2}\left(\frac{d^{2}}{2 R^{2}}-\frac{d \pi}{R}+\frac{\pi^{2}}{3}\right),
$$

which is the four dimensional $t$-channel propagator plus a correction term whose sign and magnitude depends on the fermion separation. For small separation $d<\pi R(1-1 / \sqrt{3})$ the correction enhances the magnitude of the amplitude, while for larger separation it reduces it.

It is also instructive to expand the propagator in exponentials (ignoring the mass $m$ )

$$
P_{d}(t)=-\frac{\pi R}{\sqrt{-t}}\left(e^{-\sqrt{-t} d}+e^{\sqrt{-t}(d-2 \pi R)}\right)\left(1+e^{-\sqrt{-t} 2 \pi R}+e^{-\sqrt{-t} 4 \pi R}+\ldots\right)
$$

which can be understood as a sum of contributions from five dimensional propagators. The two terms in the first parenthesis correspond to propagation from $x_{q}$ to $x_{l}$ in clockwise and counter-clockwise directions, and the series in the other parenthesis adds the possibility of also propagating an arbitrary number of times around the circle.

For later use we note that the expression for the $u$-channel KK-tower propagator $P_{d}(u)$ is identical to eq. (3. $\left.\overline{4}_{1}\right)$ with the obvious replacement $t \rightarrow u$, and $P_{d}(s)$ is obtained by analytic continuation

$$
P_{d}(s)=\frac{\pi R}{\sqrt{s-m^{2}}} \frac{\cos \left[(d-\pi R) \sqrt{s-m^{2}}\right]}{\sin \left[R \pi \sqrt{s-m^{2}}\right]} .
$$

The poles at $\sqrt{s-m^{2}}=n / R$ are not physical and can be avoided by including a finite width $\Gamma$. Note also that for $s \ll R^{-2}$ (but $s>m^{2}$ ) the relative sign between the SM propagator and it's first correction is opposite to the corresponding sign in the $t$ channel exchange case. Namely for small (large) separation the amplitude is smaller (larger) than the SM one.

Armed with this propagator it is easy to evaluate any KK boson exchange diagram in terms of its SM counterpart. For example, a pure $t$ channel exchange diagram becomes

$$
\mathcal{M}=\left(t-m^{2}\right) P_{d}(t) \times\left.\mathcal{M}\right|_{S M}
$$

where $\left.\mathcal{M}\right|_{S M}$ is the SM amplitude and the factor $\left(t-m^{2}\right) P_{d}(t)$ replaces the SM gauge boson propagator $1 /\left(t-m^{2}\right)$ by the $5 d$ propagator $P_{d}(t)$. 
We now compute $P_{d}^{f w}(t)$, the propagator between fermions which have a finite width in the extra dimension. The result is most easily obtained by integrating the propagator eq. (

$$
P_{d}^{f w}(t)=\int d y d y^{\prime}\left|f_{q}(y)\right|^{2} P_{\left|y-y^{\prime}\right|}(t)\left|f_{l}\left(y^{\prime}\right)\right|^{2}
$$

where $f_{q}\left(f_{l}\right)$ is the quark (lepton) wave function. For demonstration, we perform the integrations for the special case of Gaussian wave functions

$$
f_{f}(y)=\frac{1}{\pi^{1 / 4} \sigma^{1 / 2}} e^{-\left(y-x_{f}\right)^{2} /\left(2 \sigma^{2}\right)}
$$

as in the model of [is]. We assume that the wave functions are narrow compared to their separation and have common width. We present below the result in two relevant limits. In both cases we assume $\sqrt{-t} \gg R^{-1}$ (and therefore also neglect $m$ ). In the first case, in an intermediate momentum regime we find

$$
P_{d}^{f w}(t)=e^{-t \sigma^{2} / 2} P_{d}(t) \quad \text { for } \quad \sqrt{-t} \ll d / \sigma^{2} .
$$

Not surprisingly, the amplitude is still exponentially suppressed, but it is enhanced relative to the delta function approximation by a factor which is significant for momenta large compared to the inverse width. For much larger $t$ we find

$$
P_{d}^{f w}(t)=\frac{-\sqrt{2 \pi} R}{\sigma t} e^{-d^{2} /\left(2 \sigma^{2}\right)} \quad \text { for } \quad \sqrt{-t} \gg d / \sigma^{2}
$$

In that limit the scattering is dominated by direct local scattering through the small but non-vanishing overlap of the fermion wave functions. The propagator has the normal $4 \mathrm{~d}$ momentum dependence but the coupling is suppressed by the exponentially small wave function overlap. Since the energies attainable at upcoming colliders do not allow us to probe distances shorter than the fermion wave function width the corrections to the propagator of eq. ( $\left(\bar{A}_{1}\right)$ can be ignored for all practical purposes.

\section{B. $n$ extra dimensions}

In the case of $n>1$ extra dimensions of equal radius $R$, a straightforward extension of the above tells us that the propagator is that of the Yukawa propagator in $n$ (compact) dimensions. Let the separation be a vector $d_{i}$. If $d \equiv\left|d_{i}\right| \ll R$ and $\sqrt{-t} \gg 1 / R$, then the effects of the compactness of the space are negligible and we find the KK-tower propagator by a simple Fourier transform of the momentum space propagator

$$
P_{d_{i}}^{0}(t)=\int_{-\infty}^{\infty} d^{m} p \frac{e^{i d_{i} p_{i} / R}}{t-p_{i}^{2} / R^{2}} .
$$


The result is just the volume $(2 \pi R)^{n}$ times the Yukawa potential in the $n$ transverse dimensions, with mass $\sqrt{-t}$

$$
P_{d_{i}}^{0}(t)=-\frac{(2 \pi R)^{n}}{(2 \pi)^{n / 2}}\left(\frac{\sqrt{-t}}{d}\right)^{(n-2) / 2} K_{(n-2) / 2}(\sqrt{-t} d),
$$

where $K_{p}$ is the modified Bessel function. For large $\sqrt{-t} d$, we use the large argument limit of the Bessel function to see the exponential suppression explicitly

$$
P_{d_{i}}^{0}(t) \rightarrow-\left(\frac{2 \pi R^{2} \sqrt{-t}}{d}\right)^{(n-1) / 2} \frac{\pi R}{\sqrt{-t}} e^{-\sqrt{-t} d} .
$$

Including the effects of the finite size $R$ of the dimensions is easily done using the method of images,

$$
P_{d_{i}}(t)=\sum_{k_{i}=-\infty}^{\infty} P_{d_{i}+2 \pi k_{i} R}^{0}(t),
$$

generalizing eq. ( $\left(\overline{3}_{1}\right)$. While this sum is not given by a simple closed form expression as in the case $n=1$, for all practical purposes only the first few images make a significant contribution.

There is an important feature for the case of two or more extra dimensions that deserves comment here. For unseparated fermions, the sum over tree-level exchange of KK gauge bosons is found to be UV divergent; the relevant sum is of the form

$$
\sum_{n_{i}} \frac{1}{t-\left(n_{i} / R\right)^{2}} \sim R^{n} \int d^{n} k \frac{1}{t-k^{2}},
$$

which is clearly UV divergent for $n \geq 2$, reflecting the singularity of the Yukawa potential at short distances in two or more dimensions. This is usually dealt with by cutting the sum off at the fundamental scale $M_{*}$, but there is considerable uncertainty in doing this [i. It is easy to see that when the gauge boson exchange is between fermions separated in the extra dimensions, the separation acts as a natural cutoff and allows an unambiguous result to be obtained. The result is just given by replacing the SM propagator with $P_{d_{i}}$, which is manifestly finite. The usual UV divergence is seen in the singularity of $P_{d_{i}}$ as $d_{i} \rightarrow 0$.

Note that even in the absence of fermion separation, the width of the fermion wave function acts as a natural UV cutoff. Indeed, the integrand of eq. ( $\overline{3} .18)$ should be multiplied by

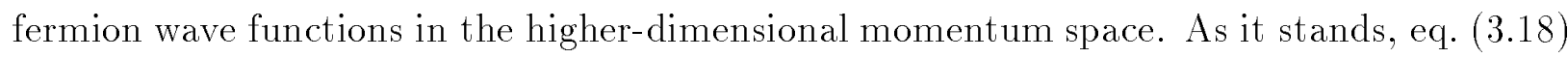
corresponds to delta function wave functions in position space. Replacing the delta functions with Gaussians of width $\sigma$ cuts off the UV divergence of eq. (3.1.18 Explicitly we calculate the leading term for small $\sigma$ (and $d=0$ ) using eqs. (

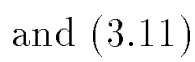

$$
\begin{array}{cc}
P_{0}^{f w}(t) \approx-\frac{(2 \pi)^{n / 2}}{n-2} \frac{R^{n}}{\sigma^{n-2}} & n>2 \\
P_{0}^{f w}(t) \approx 2 \pi R^{2} \log (\sqrt{-t} \sigma) & n=2 .
\end{array}
$$


These expressions are similar to the hard cut off results [i] 1 in, with $\sigma^{-1}$ playing the role of the cut off scale $M_{S}$.

\section{COLLIDER SIGNATURES}

Having calculated the $5 d$ propagator, the calculation of differential cross sections is a simple generalization of SM results. The general SM results can be found in Ref. [i] compute the differential cross section for deep inelastic scattering we sum over contributions from neutral current exchange (photon and $Z$ plus KK towers) $)_{-1}^{n}$ between the electron and all partons of the proton. Happily, each term in the sum is simply equal to the SM term times $t P_{d}(t)$ which can be factored so that our final expression for the differential cross section of deep inelastic scattering becomes

$$
r_{\sigma}^{t} \equiv \frac{d \sigma / d t}{d \sigma /\left.d t\right|_{\mathrm{SM}}}=\left|t P_{d}(t)\right|^{2},
$$

where $P_{d}(t)$ is given in eq. (3..4. electron momentum squared. The effect of the KK tower would be seen as a dramatic reduction of the cross section at large $-t=Q^{2}$. To illustrate this point in Fig. 1 we plot the ratio $r_{\sigma}^{t}$ of eq. ( $\left(1_{1}\right)$ as a function of $t$ for $R=1 \mathrm{TeV}^{-1}$ and representative values of $d$.

While an exponential suppression of the cross section would be an unambiguous signal of fermion separation in the extra dimension, we can still probe $d$ if a small deviation of

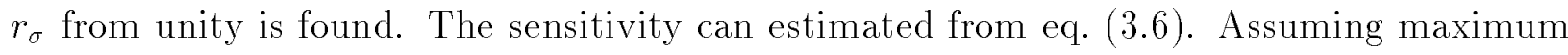
separation, $d=\pi R$, there is a reduction in the cross-section $\left(r_{\sigma}^{t}<1\right)$, and we obtain a sensitivity

$$
R \leq \sqrt{\frac{3 \Delta r_{\sigma}^{a}}{\pi^{2} Q^{2}}}
$$

where $\Delta r_{\sigma}^{a}$ is the combined theoretical and experimental error on $r_{\sigma}^{a}$. For $d=0$ one should find $r_{\sigma}^{t}>1$ with a factor of $\sqrt{2}$ higher sensitivity. At HERA, which is the only $e-p$ machine at present, we have $\Delta r_{\sigma}^{t}$ at the few percent level. Thus, we cannot obtain a strong bound from the HERA data. In the future a more energetic machine may be built. In the most optimistic scenario that is being discussed we may expect a machine with $\Delta r_{\sigma}^{t} \approx 10 \%$ at a maximum $Q^{2} \approx(4 \mathrm{TeV})^{2}$ which will be able to probe down to $R \approx(18 \mathrm{TeV})^{-1}$.

Let us now switch gears and consider the predictions of our model for high energy $e^{+} e^{-}$ or $\mu^{+} \mu^{-}$machines. The doublet and singlet components of the charged leptons may be split by a distance $d$ in the extra dimensions. This would naturally suppress the Yukawa

\footnotetext{
${ }^{\ddagger}$ In the formulae in this section we neglect $m_{Z}$. It is easy to reintroduce it, and in our numerical plots we keep it.
} 
couplings of the leptons and might be the origin of the hierarchy $m_{e} / m_{\text {top }}$ [8]. In this case the wave functions of the fermions cannot be arbitrarily narrow as the Yukawa coupling is proportional to the overlap of the wave functions of the doublet and singlet fermion. The finite width of the wave functions ultimately cuts off the exponential suppression of $t$-channel scattering amplitudes as discussed at the end of the previous section. This cut-off is somewhat model-dependent as it depends on the shape of the fermion wave functions. But if the separation of left and right handed fields is responsible for at least part of the suppression of the muon and electron Yukawa couplings then we can safely ignore the finite width of the wave functions at energies relevant to experiments.

Again, to obtain any amplitude, we simply replace all SM gauge boson propagators by their corresponding $5 \mathrm{~d}$ propagators eqs. (3..4. contributions in one channel $(s, t$ or $u$ ), then it is given by the SM cross section multiplied by the corresponding ratio of propagators as in the case of electron proton collisions. A particularly clean measurement of $d$ would be possible at a lepton collider with polarizable beams, as we could study $l_{L}^{+} l_{R}^{-} \longrightarrow l_{L}^{+} l_{R}^{-}$to isolate $t$ channel exchange. In that case the deviation from the SM predictions is given by eq. ( $\left.\left(\begin{array}{l}4 \\ 4\end{array}-\right]_{1}^{1}\right)$.

We can get more information by combining the above with the processes $e_{N}^{+} e_{N}^{-} \rightarrow \mu_{N}^{+} \mu_{N}^{-}$ ( $N=L$ or $R$ ). (The same considerations also apply to scattering into quark pairs, but this case is more difficult to study experimentally.) This process is a pure $s$ channel between unseparated fermions so that

$$
r_{\sigma}^{s N} \equiv \frac{d \sigma / d t}{d \sigma /\left.d t\right|_{\mathrm{SM}}}=\left|s P_{0}(s)\right|^{2} .
$$

For $\sqrt{s}$ small compared to the inverse size of the extra dimension the cross section is reduced independently of $d$. An extra dimensional theory without fermion separation predicts $r_{\sigma}^{s N}<$ 1 and $r_{\sigma}^{t}>1$. Thus, a measurement of $r_{\sigma}^{s N}<1$ together with $r_{\sigma}^{t}<1$ would be evidence for fermion separation in the extra dimension.

Another interesting probe of $d$ using $s$ channel has been suggested recently [i] ${ }_{-1}^{-1}$. Suppose that the first KK mode has been produced and its mass $1 / R$ measured. The case of $d=0$ can be distinguished from $d \neq 0$ by looking at the cross-section at lower energies. In particular, for $d=0$, the first KK exchange exactly cancels the SM amplitude at $\sqrt{s}=1 /(\sqrt{2} R)$, whereas for $d \neq 0$ the cross-section can still be large. Therefore, a beam scan at energies beneath the first resonance can be an efficient probe of $d$.

Even if beam polarization is not available, one can still probe the nature of the extra dimensions by looking at several processes and using angular information. First consider an unpolarized $e^{+} e^{-} \rightarrow \ell^{+} \ell^{-}$scattering. (The same holds for incoming muons.) We get the tree level cross section

$$
\frac{d \sigma}{d t}=\frac{\pi \alpha^{2}}{s^{2}}\left[\left(1+\frac{1}{16 \sin ^{4} \theta_{w}}\right) \frac{u^{2}\left(P_{0}(s)+P_{0}(t)\right)^{2}}{\cos ^{4} \theta_{w}}+\frac{t^{2} P_{d}^{2}(s)+s^{2} P_{d}^{2}(t)}{2 \cos ^{4} \theta_{w}}\right] .
$$

When $\ell=e$ both $s$ and $t$ channels are possible, while for $\ell \neq \epsilon$ only the $s$ channel is present, and in the above formula one should set $P_{d}(t)=P_{0}(t)=0$. We also define, as before, the 
ratio of the $5 \mathrm{~d}$ cross section to the $\mathrm{SM}$ one as $r_{\sigma}^{s}\left(r_{\sigma}^{s t}\right)$ for the $e^{+} e^{-} \rightarrow \mu^{+} \mu^{-}\left(e^{+} e^{-} \rightarrow e^{+} e^{-}\right)$ reaction. In Figs. 2 and 3, we presented $r_{\sigma}^{s}$ and $r_{\sigma}^{s t}$ as a function of the scattering angle. As we can see, the cross sections depend in a non trivial way on the separation. This is because the helicity changing amplitude depends on $d$, while the helicity conserving one does not. By looking at angular distributions, one can separate the different contributions, and extract both $R$ and $d$.

Another interesting collider mode which allows a very clean measurement of fermion separations is $e^{-} e^{-}$scattering. The advantage of the $e^{-} e^{-}$mode is that both beams can be polarized to a high degree which allows for a clean separation of the interesting $t$ and $u$ channels from $s$ channel. We find for $e_{L}^{-} e_{R}^{-}$scattering to $e^{-} e^{-}$(summed over final polarizations)

$$
r_{\sigma}^{t u} \equiv \frac{d \sigma / d t}{d \sigma /\left.d t\right|_{\mathrm{SM}}}=\frac{u^{2}\left|P_{d}(t)\right|^{2}+t^{2}\left|P_{d}(u)\right|^{2}}{u^{2} / t^{2}+t^{2} / u^{2}} .
$$

Last, we estimate the sensitivity of lepton colliders. Assuming $\Delta r_{\sigma} \approx 1 \%$ and using

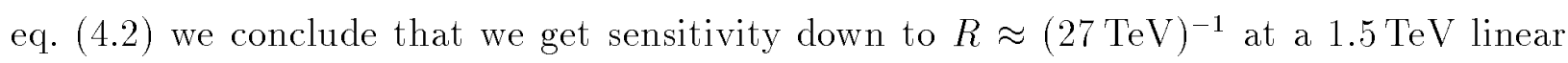
collider and $R \approx(72 \mathrm{TeV})^{-1}$ at a $4 \mathrm{TeV}$ muon collider.

A hadron machine could also be used to probe extra dimensional separations. Here, the situation is somewhat more complicated as there are many subprocesses that contribute, the theoretical predictions are more uncertain and the experimental situation is more complicated. However, the higher energy of the hadron machine compensates for these drawbacks.

One possible probe is to look into dijet production, in particular, for high $p_{T}$ jets. This process occurs via $q q, q \bar{q}$ and $g g$ scattering that occurs via $s, t$ and $u$ channels. In our framework the first two will be modified in a way similar to what we described for the leptons. In general, the invariant mass of the two jets can be measured and thus one can find $\hat{s}$, the parton center of mass of the event. Combining it with the angular information one can determine both $s$ and $t$ for each event. This double differential cross section is sensitive to the size of the extra dimension and the fermion separation. Another possible probe of our scenario is Drell-Yan processes. Here, while one has less statistics, the accuracy is higher. In contrast to the dijet case, this is a pure $s$ channel. Of course, for both of these cases a more detailed study needs be done to see exactly what kind of sensitivity is attainable. Assuming $\Delta r_{\sigma} \approx 10 \%$ at a maximum $Q^{2} \approx(7 \mathrm{TeV})^{2}$ and using eq. ('A.2. able to probe down to $R \approx(40 \mathrm{TeV})^{-1}$.

We have so far contented ourselves to putting limits on the model, in some cases noting that the difference between extra dimensional models with and without fermion separation could be resolved. It is more exciting to consider how large a positive signal for exponentially dropping cross-sections could reasonably be expected at future colliders. The direct limits from searching for the KK gauge bosons (and $Z^{\prime}$ searches) imply $1 / R \geq 800 \mathrm{GeV}$. On the other hand, precision electroweak bounds on higher-dimensional operators generated by KK exchange place a far more stringent limit $1 / R \gtrsim 3 \mathrm{TeV}\left[\underline{1} \overline{2}_{1}\right]$. If we take these precision bounds seriously, then a $1.5 \mathrm{TeV}$ NLC could still observe a drop in the cross-section by as much as 
a factor of 2 for backscattering. However a $4 \mathrm{TeV}$ muon collider could see a reduction by as much as a factor of 60 . More optimistically, we can imagine that there are extra states in the bulk whose exchange modifies the precision electroweak analysis. If these bounds are ignored, the direct bounds are weak enough that spectacular drops in the cross-section can be observed, by as much as a factor of 1000 at a $1.5 \mathrm{TeV}$ NLC.

\section{DISCUSSION}

Our signal displays a remarkable fact about scenarios with fermions split in the extra dimensions. Traditionally, when fermion fields are either delocalized in the extra dimensions or when they are localized without any splitting, at energies above the compactification scale all the amplitudes grow faster than in 4-dimensions. This reflects the non-renormalizable nature of higher-dimensional gauge theories. Here, we instead see that for $t$ and $u$ channel interactions between fermions localized at different points, the cross-section decreases exponentially. The separation acts as a physical "point-splitting" regularization of the non-renormalizable theory, allowing essentially exact computations for some amplitudes completely independent of the physics at the ultimate UV cutoff (which is smaller than the separation).

This result, that fermion separation allows us to make unambiguous predictions for some quantities in non-renormalizable theories which are exponentially insensitive to physics at the cutoff $M_{*}$, is very general. We have already discussed how fermion separation provides a physical UV cutoff for the KK gauge boson exchange. As another example, in the context of large extra dimensions with low fundamental Planck scale, several groups have considered the effects of tree-level graviton exchange in the higher dimensions [1] $\left.{ }_{1}^{1}\right]$. For two or more extra dimensions, the sum over the graviton KK excitations is UV divergent. Cutting off this divergent sum at the scale $M_{*}$ generates an operator of the form

$$
\mathcal{O}=\lambda \frac{T_{\mu \nu} T^{\mu \nu}}{M_{*}^{4}}
$$

where $T_{\mu \nu}$ is the $4 \mathrm{~d}$ energy momentum tensor, and $\lambda$ is an unknown constant dependent on the details of how the KK sum is cut off. The analysis then proceeds by examining the effect of this particular higher-dimension operator on various observables. Even if deviations consistent with this operator are seen experimentally, however, this does not provide direct evidence for extra dimensions. For instance, the operator may be generated by integrating out a single massive spin 2 particle of mass $\sim M_{*}$. On the other hand, if the quarks and leptons are split by some distance $d$, the UV divergence is automatically cut-off and we can write essentially the exact expression for the cross section of e.g., electron proton scattering including the graviton exchange. The expression will only depend on the unknowns $d$, the number of extra dimensions $n$ and the higher-dimensional Newton constant $G_{N(4+n)}$. The only in principle incalculable corrections come from the higher-dimensional operators

suppressed by $M_{*}$, but these will be suppressed by $\sim e^{-100}$ for the same reason that proton decay is suppressed to acceptable levels. 
It is also important to note that the scattering of split fermions remains small even above the scale of quantum gravity $M_{*}$, say the string scale. The reason is still the same; as long as the fermions remain localized apart from each other at these energies, all the new heavy states which come in at $M_{*}$ still need to propagate from one fermion to the other, providing a still further suppression of the amplitudes. It is interesting that in this scenario, we could in principle have the best of all worlds in super-Planckian physics. The usual expectation is that above $M_{*}$, all sorts of new physics hit us at once with a rich and (at least initially) chaotic set of signals. We retain this possibility in the $s$ channel. But in the $t$ and $u$ channels, the interactions between split fermions provide an antiseptic environment where the properties of all modes lighter than the inverse fermion separation (which can include fascinating objects such as bulk gravitons) can be unambiguously studied.

Finally, we comment on different possible physics that leads to exponentially small crosssections at large $t$, possibly faking our most dramatic signal. Consider some composite object

with some fuzzy size $\Lambda$. For $\sqrt{-t}$ smaller than $\Lambda$, we expect that the cross-sections decrease with $\sqrt{-t}$. Of course, if these are composite objects like the proton, consisting of point-like partons, then for $\sqrt{-t}>\Lambda$ we expect the usual power-law fall-off with $t$ expected from scattering off the point-like constituents, so this can not fake our signal. On the other hand, if the fuzziness is like that of a string, we may expect that the exponentially decreasing crosssections persist above $\Lambda$. However, in this case we do not expect the decreasing amplitude to have any simple relationship to the SM amplitudes, whereas for us the new cross-section is directly related to the $\mathrm{SM}$ as in e.g., eqs. (1 between the exponentially falling amplitudes with the SM ones is the smoking gun for the observation of fermion separation in extra dimensions at future colliders.

\section{ACKNOWLEDGMENTS}

We thank Stan Brodsky, Hooman Davoudiasl, Lance Dixon, Hitoshi Murayama and Tom Rizzo for useful discussions. N.A.-H. is supported by DOE under contract DE-AC0376SF00098 and by NSF under contract PHY-95-14797. Y.G. and M.S. are supported by the Department of Energy under contract DE-AC03-76SF00515. 


\section{REFERENCES}

[1] N. Arkani-Hamed, S. Dimopoulos and G. Dvali, Phys. Lett. B429 (1998) 263; Phys. Rev. D59 (1999) 086004; I. Antoniadis, N. Arkani-Hamed, S. Dimopoulos and G. Dvali, Phys. Lett. B436 (1998) 257; N. Arkani-Hamed, S. Dimopoulos and J. March-Russell, hep-th/9809124,

[2] N. Arkani-Hamed and S. Dimopoulos, hep-ph

[3] See also E. Witten, Nucl. Phys. B471 (1996) 135; P. Horava and E. Witten, Nucl. Phys. B460 (1996) 506 and Nucl. Phys. B475 (1996) 94; J. Lykken, Phys. Rev. D54 (1996) 3693; G. Shiu and S.-H.H Tye, Phys. Rev. D58 (1998) 106007; R. Sundrum, Phys. Rev. D59 (1999) 085009; Z. Kakushadze and S.-H.H. Tye, Nucl. Phys. B548 (1999) 180.

[4] K. Dienes, E. Dudas and T. Gherghetta, Phys. Lett. B436 (1998) 55; Nucl. Phys. B537 (1999) 47.

[5] L. Randall and R. Sundrum, thep-ph [9905221: and hep-th]9906064:

[6] N. Arkani-Hamed, S. Dimopoulos, G. Dvali and N. Kaloper, hep-th/99007209; J. Lykken and L. Randall, "hep-th/9908076.

[7] L. Ibanez and G. Ross, Nucl. Phys. B368 (1992) 3.

[8] N. Arkani-Hamed and M. Schmaltz, hep-ph [9903417.

[9] S.C. Bennett, C.E. Wieman, Phys. Rev. Lett. 82 (1999) 2484.

[10] N. Arkani-Hamed, L.J. Hall and M. Schmaltz, to appear.

[11] E.A. Mirabelli and M. Schmaltz, to appear.

[12] T.G. Rizzo and J.D. Wells, hiep-ph 2906234 ;. T. Rizzo, private communication.

[13] T.G. Rizzo, hepep-ph $\overline{9} \overline{9} \overline{0} \overline{9} \overline{2} \overline{3} \overline{2}_{\text {r }}^{\prime}$

[14] T. Han, J. Lykken and R-J. Zhang, Phys. Rev. D59 (1999) 105006.

[15] V.D. Barger and R.J.N. Phillips, Collider Physics. Addison-Wesley, 1996.

[16] See for instance G.F. Giudice, R. Rattazzi, J.D. Wells, Nucl. Phys. B544 (1999) 544; J.L. Hewett, Phys. Rev. Lett. 82 (1999) 4765; H. Davoudiasl, ihep-ph 29904425. 


\section{FIGURES}

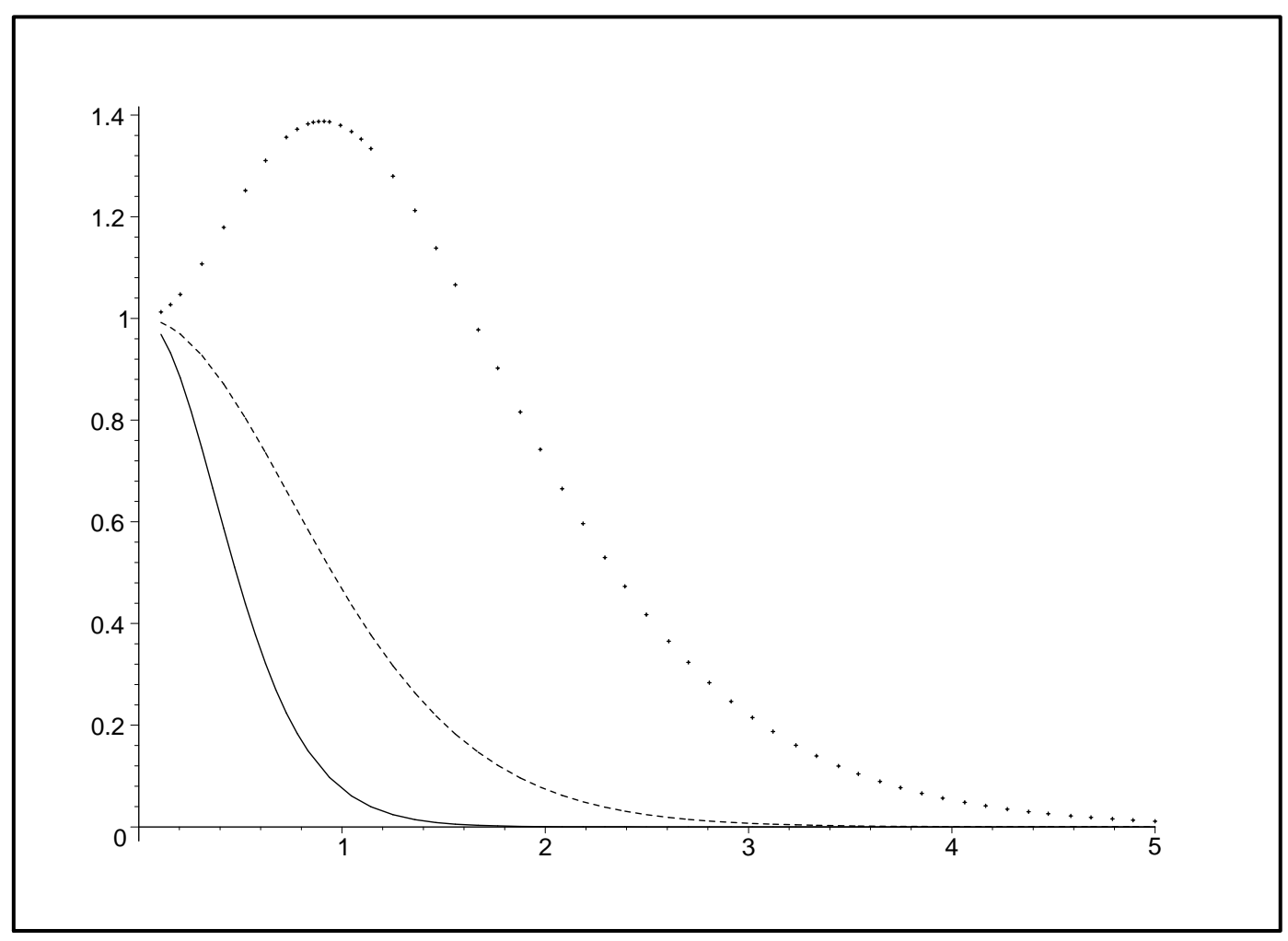

FIG. $1 . r_{\sigma}^{t}$ (the cross section for $t$ channel exchange in the $5 \mathrm{~d}$ theory normalized by the corresponding SM cross section) as a function of $\sqrt{-t}$ in units of TeV. We assume $R^{-1}=1 \mathrm{TeV}$. The dotted, dashed and solid curves are for separations of $d / R=1, \pi / 2$ and $\pi$ respectively. 


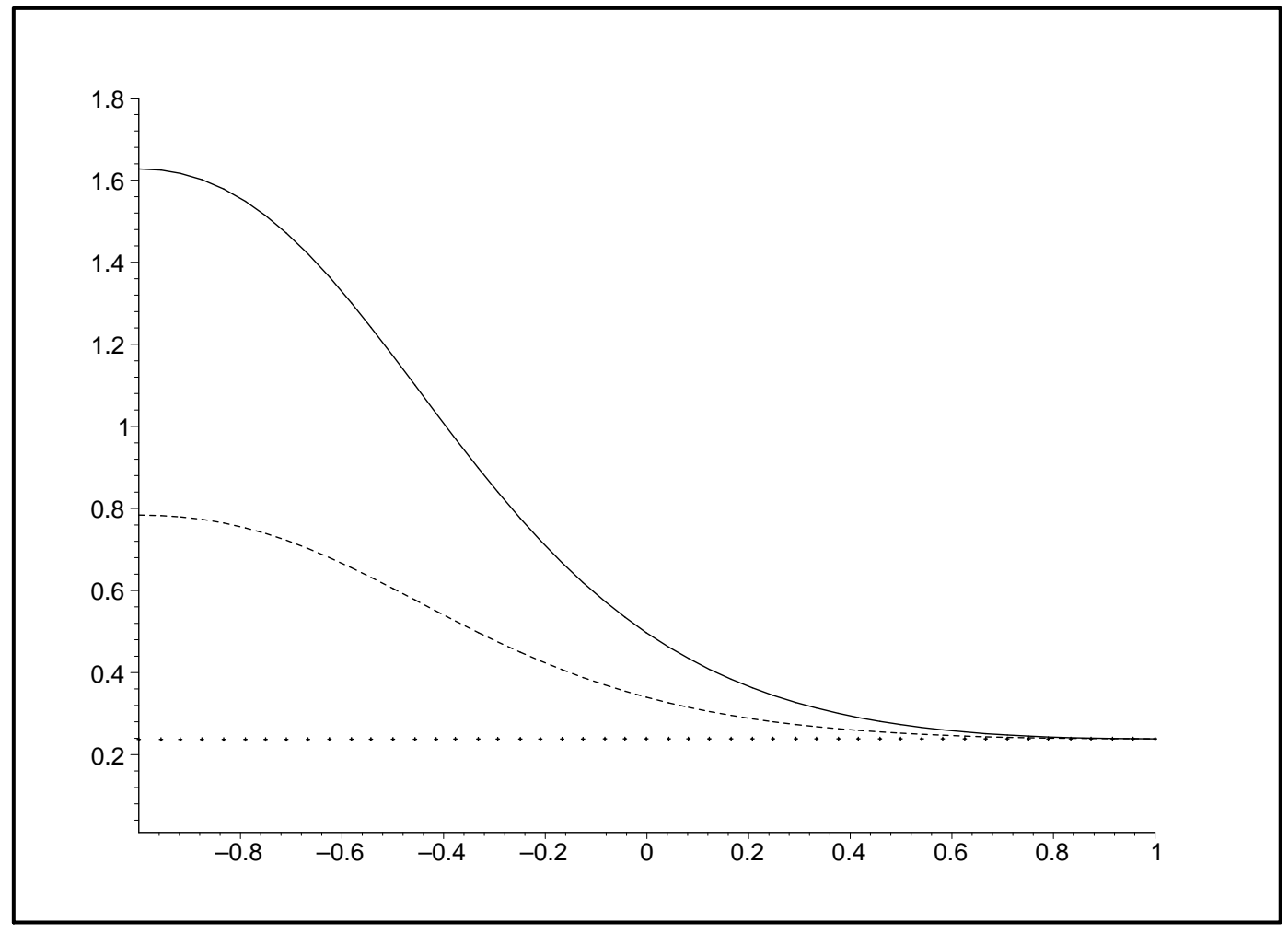

FIG. 2. $r_{\sigma}^{s}$ (the cross section for $s$ channel exchange, e.g. $e^{+} e^{-} \rightarrow \mu^{+} \mu^{-}$, in the $5 d$ theory normalized by the corresponding SM cross section) as a function of the scattering angle, $\cos \theta$. We assume $R^{-1}=4 \mathrm{TeV}$ and $\sqrt{s}=1.5 \mathrm{TeV}$. The dotted, dashed and solid curves are for separation of $d / R=0,1$ and $\pi$ respectively. 


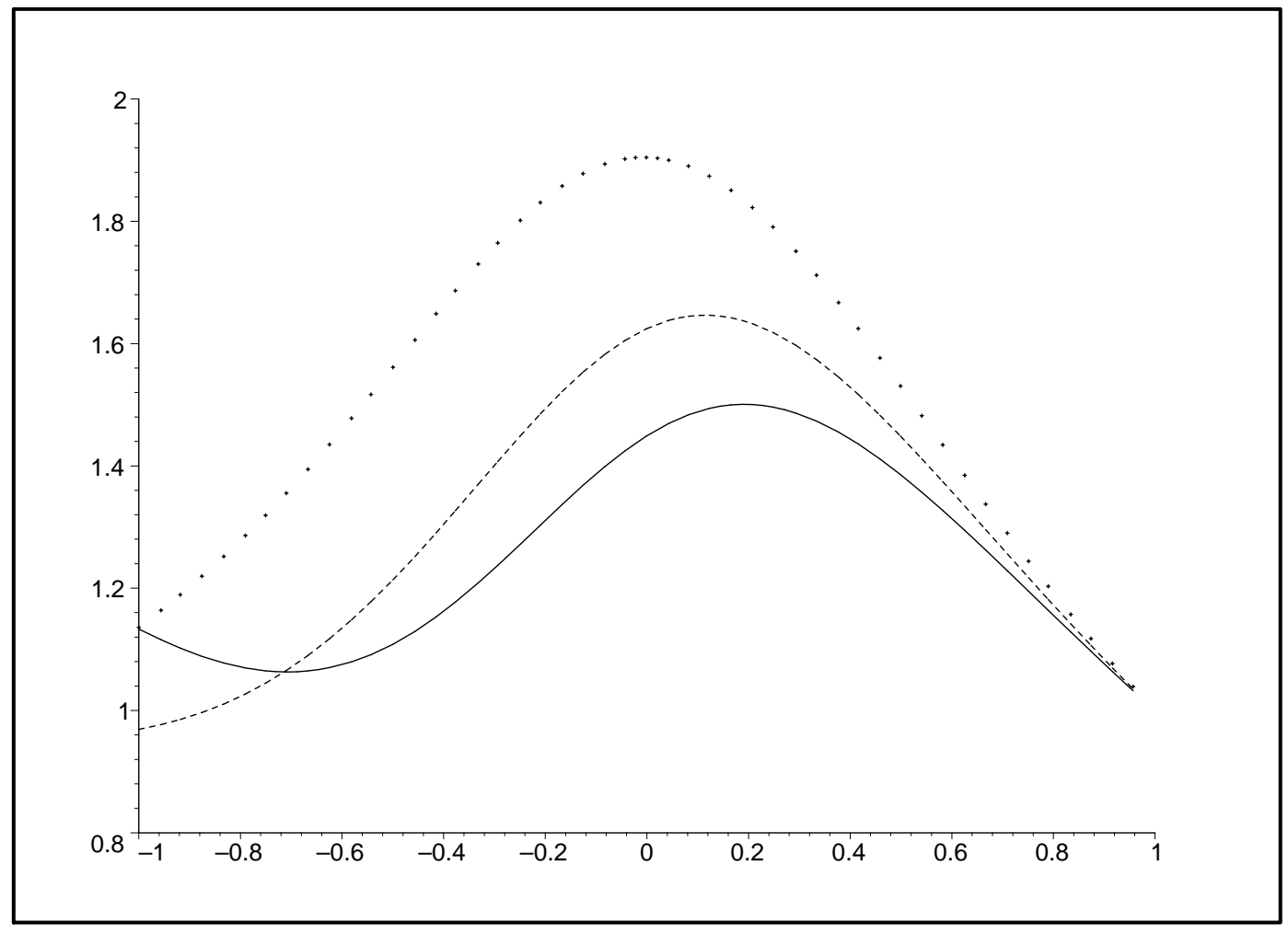

FIG. 3. Same as fig. 2 for $r_{\sigma}^{s t}$ (the cross section for $s$ and $t$ channel exchange, e.g. $e^{+} e^{-} \rightarrow e^{+} e^{-}$, in the $5 \mathrm{~d}$ theory normalized by the corresponding SM cross section). 